\title{
Prevalence of dementia among widowed and non-widowed patients and associated clinical and sociodemographic characteristics
}

\author{
Rodrigo Rizek Schultz' \\ Paulo Eduardo Lahoz Fernandez' \\ Neil Ferreira Novo² 1 D \\ Yara Juliano ${ }^{2}$ (D) \\ José Roberto Wajman' ${ }^{1}$
}

\section{Abstract}

Objective: to verify if the prevalence of dementia differs between widowed and nonwidowed elderly persons and between genders, and to analyse if there is an association with sociodemographic and clinical characteristics. Method: a retrospective cross-sectional observational study of patients treated at a Behavioral Neurology outpatient clinic from 1999 to 2009 was carried out, employing anamnesis, physical and neurological examination, the Clinical Dementia Rating Scale (CDR) and the Mini Mental State Examination (MMSE). Sociodemographic (schooling and age) and clinical (age of onset of symptoms and time since onset of symptoms, MMSE and CDR) variables were analyzed. The differences were evaluated by the Mann Whitney test, using a significance value of $p<0.05$. Results: of 208 patients diagnosed with dementia, $73(35.1 \%)$ were widowed and $135(64.9 \%)$ were non-widowed. Those who were widowed were older than those who were non-widowed $(p<0.001)$ when diagnosed with dementia. This difference in age remained when gender $(p<0.001)$, widowed and widowed women $(p<0.001)$ and widowed and non-widowed men $(p<0.001)$ were compared. The time from onset of symptoms to diagnosis was greater in widowed than in non-widowed men $[55.6( \pm 86.3)$ vs. 43.4 $( \pm 44.8)$ months], although the difference was not statistically significant. Widowed patients with dementia had lower schooling, regardless of gender $(p<0.05)$. Conclusion: the prevalence of dementia differed between widowed and non-widowed individuals, being higher among non-widows. There was an association between widowhood and the clinical and sociodemographic characteristics, with differences between the genders. The loss of a spouse can generate different outcomes among men and women, necessitating measures with a specific focus on prevention and strategies of care in dementia.

\footnotetext{
Universidade de Santo Amaro, Setor de Neurologia do Comportamento, Departamento de Clínica Médica. São Paulo, São Paulo, Brasil.

2 Universidade de Santo Amaro, Curso de Pós-graduação em Ciências da Saúde. São Paulo, São Paulo,
} Brasil.
Keywords: Dementia. Alzheimer's Disease. Epidemiology. Widowhood. 


\section{INTRODUCTION}

The relationship between dementia and widowhood has been recently analyzed. Evidence shows that marital status can prevent the risk of dementia, through daily social interactions, together with the enhancement of cognitive reserves, the development of coping skills, and increased resilience ${ }^{1}$.

Data suggests that marriage can result in greater social contact, which in turn can lead to a lower risk of developing dementia ${ }^{2}$. However, in contrast, the loss of a spouse can bring additional stress through grieving, and can therefore facilitate the occurrence of dementia ${ }^{3}$.

Studies show that widowhood can indeed reduce stress, through a reduced burden upon the caregiver and the reduced exposure of the spouse to suffering, representing a factor of protection against forms of dementia ${ }^{4}$. Social relationships should be taken as a factor that brings considerable risk, and socially motivated interventions are factors that could provide an opportunity to reduce overall risk ${ }^{5}$.

Even though there some studies have established a connection between dementia and widowhood, there remains a lack of evidence in specialized literature regarding the prevalence and the social and demographic profiles of those individuals, both widowed and non-widowed, as well as the impact of widowhood on different genders. The purpose of the study was to assess if there is a difference in the prevalence of dementia between widowed and non-widowed individuals; to consider whether there is any association with other social, demographic and clinical characteristics and, at the same time, verify if there are any differences between the genders.

\section{METHOD}

All individuals involved in the study were evaluated at the Behavioral Neurology Sector of the Universidade de Santo Amaro, in the city of São Paulo, São Paulo state, Brazil. This study was accepted by the Research Ethics Committee under registration number $128 / 2015$. The patients who were treated at this outpatient unit either sought treatment, or were referred due to cognitive disorders or behavioral disorders which suggested some type of neuropsychiatric disorder. As a criterion for inclusion, cases with first diagnosis of dementia as defined on the medical records were accepted. Patients with other types of cognitive disorder were excluded, as well as those who did not meet the criteria adopted for the definition of dementia.

A cross-sectional observational study was performed based on the analysis of 297 medical records, selected based on one sole criterion, that of being duly completed and containing comprehensive information about patients who had received outpatient treatment between 1999 and 2009 , corresponding to the period over which the proposed services were provided. The individuals selected for appraisal were part of the Behavioral Neurology Department of the Universidade de Santo Amaro, and were served by public health services in the Greater São Paulo metropolitan region. The treatment and the collection of information were both performed by the same neurologist, and the cognitive and functional analysis was performed through the Mini Mental State Exam (MMSE) and also through the Clinical Dementia Rating Scale (CDR), applied by a psychologist specializing in neuropsychology $y^{6,7}$. The data was analyzed to see if there was a difference between the number of patients with dementia, between the two groups considered (widowed and non-widowed), considering social and demographic (schooling and age, respectively) and clinical variables (age at onset of symptoms, time since onset of symptoms, MMSE and $\mathrm{CDR}$ ) variables. There was also an appraisal as to whether there was any social, demographic or clinical differences between men and women, considering both widowed and non-widowed individuals, and if there was a difference in gender among widowed and non-widowed people with dementia. No distinction was made between single people, those living with a partner, and those who were separated. To appraise dementia and differences between genders, the sample was divided into two groups: women with dementia and men with dementia. In addition, there was a separate analysis of differences between the genders based on the comparison of widowed and non-widowed women, as also between widowed and non-widowed men. 
The diagnosis of dementia was based on the criteria set out in the Diagnostic and Statistical Manual of Mental Disorders - Fourth Edition (DSMIV $)^{8}$. The clinical diagnosis was made according to the following published criteria: Those of the National Institute of Neurological Communicative Disorders and Stroke-Alzheimer's Disease and Related Disorders Association (NINCDS-ADRDA) for Alzheimer's Disease ${ }^{9}$, and of the National Institute of Neurological Disorders and Stroke - Association Internationale pourla Recherche et L'Enseignement en Neurosciences (NINDSAIREN) for vascular dementia (hereinafter DV) ${ }^{10}$.

The Mann-Whitney test was applied to compare the groups with and without dementia, according to their gender and widowhood status. The MannWhitney test was also performed for each of the following variables: age; age at onset of symptoms, time since onset of symptoms (in months), schooling (in years), MMSE and CDR. For statistical analysis, a specific statistics programme was used. The level of significance was set at $p<0.05$.

\section{RESULTS}

A total of $208(70.03 \%)$ patients with dementia and $89(29.96 \%)$ without dementia were identified. The group of people without dementia included those with a diagnosis of subjective cognitive impairment and those showing some kind of psychological or psychiatric disorder; however, the diagnosis was not defined. Of the group with dementia, 73 patients (35\%) were widowed, while 135 (65\%) were not. It was found that widowed patients with dementia were older $(p<0.0001)$ and had a lower level of schooling $(p<0.0001)$, than those who were not widowed (Table 1).

In relation to gender, women with dementia tended to be older $(p<0.0001)$ and have a lower level of schooling $(p=0.0008)$ than men (Table 2$)$.

In addition, widows were older $(p=0.0001)$ and had a lower level of schooling ( $p=0.0387$ ) (Table 3). Similarly, widowers tended to be older $(p=0.0005)$ and have a lower level of schooling $(p=0.0340)$ (Table 4).

Table 1. Difference between widowed $(n=73)$ and non-widowed patients $(n=135)$ living with dementia. São Paulo, SP, 1999-2009.

\begin{tabular}{llll}
\hline Variables & Widowed & Not widowed & Widowed x Not-Widowed \\
& Mean $( \pm \mathrm{SD})$ & Mean $( \pm \mathrm{SD})$ & $p$ \\
\hline Age & $78( \pm 12)$ & $67 \pm 10)$ & $<0.0001$ \\
Schooling (years) & $1.89( \pm 2.53)$ & $3.54( \pm 3.52)$ & $<0.0001$ \\
Age at onset of symptoms & $74.3( \pm 8)$ & $69.3( \pm 12.9)$ & $<0.0001$ \\
Time since onset of symptoms (months) & $46( \pm 48.5)$ & $43.1( \pm 58.1)$ & 0.2169 \\
Mini Mental State Exam & $10.5( \pm 7.67)$ & $14.7( \pm 9.24)$ & $<0.0001$ \\
Clinical Dementia Rating & $2.12( \pm 0.74)$ & $1.91( \pm 0.81)$ & 0.0446 \\
\hline
\end{tabular}

The $p$ value refers to the Mann-Whitney test. $\mathrm{SD}=$ Standard Deviation.

Table 2. Difference between women $(n=98)$ and men $(n=110)$ living with dementia. São Paulo, SP, 1999-2009.

\begin{tabular}{llll}
\hline Variables & Women & Men & Women $x$ Men \\
& Mean $( \pm \mathrm{SD})$ & Mean $(\underline{\mathrm{SD}})$ & $p$ \\
\hline Age & $75.3( \pm 9.7)$ & $67.5( \pm 13)$ & $<0.0001$ \\
Schooling (years) & $2.1( \pm 2.5)$ & $3.7( \pm 3.7)$ & 0.0008 \\
Age at onset of symptoms & $71.6( \pm 9.9)$ & $63.9( \pm 13.3)$ & $<0.0001$ \\
Time from start of symptoms (months) & $44.2( \pm 31.2)$ & $44.3( \pm 33.3)$ & 0.3893 \\
Mini Mental State Exam & $10.8( \pm 6.6)$ & $15.3( \pm 7.5)$ & $<0.0001$ \\
Clinical Dementia Rating & $2.17( \pm 0.77)$ & $1.81( \pm 0.76)$ & 0.0018 \\
\hline
\end{tabular}

The $p$ value refers to the Mann-Whitney test. SD = Standard Deviation. 
Table 3. Difference between widows $(n=58)$ and non-widowed women $(n=40)$ living with dementia, São Paulo, SP, 1999-2009.

\begin{tabular}{llll}
\hline Variables & Widows & Non-widowed & Widows x Non-widows \\
& Mean $( \pm \mathrm{SD})$ & Mean $( \pm \mathrm{SD})$ & $p$ \\
\hline Age & $78.3( \pm 7.4)$ & $70.4( \pm 10.5)$ & 0.0001 \\
Schooling (years) & $1.83( \pm 2.51)$ & $2.53( \pm 2.53)$ & 0.0387 \\
Age at onset of symptoms & $74.9( \pm 7.4)$ & $66.8( \pm 11.1)$ & 0.0002 \\
Time since onset of symptoms (months) & $43.3( \pm 33.3)$ & $42.5( \pm 64.3)$ & 0.0588 \\
Mini-Mental State Exam & $10.2( \pm 6.4)$ & $11.9( \pm 7.1)$ & 0.1000 \\
Clinical Dementia Rating & $2.25( \pm 0.74)$ & $2.05( \pm 0.81)$ & 0.1325 \\
\hline
\end{tabular}

The $p$ value refers to the Mann-Whitney test. SD $=$ Standard Deviation.

Table 4. Difference between widowers $(n=15)$ and non-widowed men $(n=95)$ living with dementia, São Paulo, SP, 1999-2009.

\begin{tabular}{llll}
\hline Variables & Widowers & Non-widowed men & Widowers x Non-widowed men \\
& Mean $( \pm \mathrm{SD})$ & Mean $( \pm \mathrm{SD})$ & $p$ \\
\hline Age & $76.6( \pm 8.95)$ & $66.2( \pm 13.28)$ & 0.0005 \\
Schooling (years) & $2.20( \pm 2.67)$ & $3.98( \pm 3.78)$ & 0.0340 \\
Age at onset of symptoms & $71.9( \pm 9.9)$ & $63.2( \pm 13.5)$ & 0.0043 \\
Time since onset of symptoms (months) & $55.6( \pm 86.3)$ & $43.4( \pm 44.8)$ & 0.3248 \\
Mini-mental state exam & $11.6( \pm 5.4)$ & $16.0( \pm 7.7)$ & 0.0120 \\
Clinical Dementia Rating & Not evaluated & Not evaluated & Not evaluated \\
\hline
\end{tabular}

The $p$ value refers to the Mann-Whitney test. $\mathrm{SD}=$ Standard Deviation.

With reference to the clinical variables, it was found that widowed patients living with dementia tended to have been older when the symptoms started $(p<0.0001)$, have lower MMSE scores $(p<0.0001)$ and also higher CDR scores $(p=0.0446)$. There was no statistical difference in the time since the onset of symptoms ( $p=0.2169)$ (Table 1$)$. In terms of gender, women living with dementia tended to be older at the onset of symptoms $(p<0.0001)$, have lower MMSE scores $(p<0.0001)$ and a higher CDR $(p=0.0018)$. There was no statistical difference in time since onset of symptoms $(p=0.3893)$ (Table 2$)$. Widows living with dementia were also older at the onset of symptoms than non-widows $(p=0.0002)$, but there was no statistical difference in time since onset of symptoms $(p=0.0588)$, MMSE score $(p=0.10)$ and CDR $(p=0.1325)$ (Table 3$)$. Widowers were older at the onset of symptoms $(p=0.0043)$ and had a lower MMSE score $(p=0.0120)$. There was no statistical difference in time elapsed since onset of symptoms $(p=0.3248)$ and CDR was not appraised in widowers, due to a lack of sufficient data in the medical records (Table 4).

\section{DISCUSSION}

According to the present study, people living with dementia were generally older than those without dementia, when both genders were considered together. A metanalysis published in 2018 showed that widowed people had a $20 \%$ higher risk of developing dementia than married people, in studies duly adjusted for age and gender ${ }^{11}$. These results were found in studies where dementia was diagnosed based on the clinical examination on all participants, rather than through analyses of data collected from information gathered through routine collection. This study found a greater risk of dementia developing among widowed people, in case-control 
and cross-sectional studies, compared with cohort studies ${ }^{11}$. These results confirm the findings of the present study, which exhibited a greater occurrence of dementia among widowed people, which links widowhood to greater conditions of stress and less social support for dealing with daily activities ${ }^{12}$.

The loss of a spouse is considered one of the most stress-inducing events that a person can face. Widowed individuals exhibit a greater risk of psychological disorders, and also higher mortality, than married people ${ }^{13}$. A study showed a lower risk of dementia among widowed people, compared with other categories of marital status, including unmarried, single, and divorced; however, the duration of the study was not deemed sufficient to evaluate these differences. According to the present study, social and economic factors can contribute to the differences between the genders, in combination with marital status, among cases of people living with dementia ${ }^{14}$.

It was found that widowed patients living with dementia tended to be older than non-widowed patients (Table 1). This agrees with a recent study which showed that, as time elapsed after the death of a spouse, there is a significant acceleration of the process of cognitive decline, thereby strengthening the concept that widowhood increases the risk of cognitive impairment among older individuals ${ }^{15}$.

Level of schooling was higher among nonwidowed people than widowed people living with dementia (Table 1). According to cognitive reserve theory, level of schooling and other exposures that occur throughout life, such as mental and social activities, provide alternative cognitive operations or neural networks of greater efficiency, which enable individuals to better handle brain damage, thereby delaying the onset of dementia ${ }^{16}$. These results agree with the data of previous studies that appraised the beneficial effects of marriage upon cognitive reserve and found that the resilience of the relationship is responsible for a lower occurrence of neuropathological damage ${ }^{17}$. In contrast, another study showed that caregivers may remain, or become, resilient over time, in spite of the deterioration of health, institutionalization and death of the respective spouse. This being the case, widowhood cannot always be seen as a barrier to resilience ${ }^{18}$.
The age at the onset of symptoms was higher in widowed patients living with dementia than nonwidowed patients living with dementia (Table 1). This adds weight to the theory that a patient may delay seeking medical care when he no longer has a spouse to note his symptoms. As the number of widows was greater than the number of widowers, it is possible that women may seek health care only in more advanced stages of dementia, even after the death of the spouse, resulting in a more advanced age at the onset of symptoms, than in non-widowed women with dementia.

Regarding MMSE and widowhood, widowed patients with dementia had lower scores than nonwidowed patients with dementia (Table 1). These results suggest that a marital status of widowed is associated with worse cognitive performance. A study that appraised cognitive decline through the MMSE showed that being single or widowed increased the likelihood of cognitive decline, with a greater impact among men, a result which matches the findings of the present study (Table 4) ${ }^{19}$.

The CDR values were higher among widowed people with dementia than non-widowed people living with dementia, which also suggests that the severity of the dementia is greater among widowed individuals (Table 1). There is evidence that the greater severity of dementia among married people has been favorably associated with total duration of care. However, there is a dearth of studies evaluating this kind of measure among widowed people ${ }^{20}$.

In the present study within the group of patients living with dementia, women dementia patients tended to be older than men dementia patients, a fact that agrees with other previous studies, showing that women have a longer survival period, and are also diagnosed at more advanced stages of the disease than men (Table 2$)^{21}$. In addition, women had a lower level of schooling, lower MMSE score, and higher CDR score (Table 2). It is known that dementia affects women disproportionately in comparison to men, and so health care providers should provide a better response to patient need ${ }^{22}$. This could be linked to the fact that female caregivers also need to take on new activities that in the past were carried out by the individuals with dementia, 
thereby contributing to an increase in stress and also burden among these women ${ }^{23}$. Most of these are adult daughters or spouses of patients living with dementia, aged over 65 , which makes the tasks of caring more difficult. In addition, women tend to spend more time on care activities than men ${ }^{24}$. The reasons for this are many, but one possibility could be that women are less likely to work outside the home $e^{24,25}$. Another feasible explanation is that women more often end up being caregivers due to the cultural expectations placed upon them. The fact that men with dementia are younger at the onset of symptoms, as well as having a greater MMSE score and lower CDR score, could be linked to greater care provided by their spouses, a fact which would lead men to seek treatment more quickly, after cognitive disorders were observed.

When analysing the social and demographical variables separately by gender, it was found that both widows and widowers were older and had a lower level of schooling than non-widowed men and women, with the greatest difference observed among widowers (Table 3 and 4). Age at onset of symptoms was greater in both widowed men and women; however, MMSE was only different in men, with widowers achieving lower scores. This result agrees with another study that reveals the greater impact of widowhood among men (Tables 3 and 4$)^{19}$. There was no difference in CDR scores between widowed and non-widowed women. It was not possible to evaluate this variable among men, due to the lack of sufficient data in medical records (Tables 3 and 4).

The present study had some limitations. As it is a retrospective study, no statistical corrections for age or level of schooling were made. In addition, as the differences between specific marital statuses were not considered, there is a possibility of methodological bias, as people who were single, separated, or living with a partner do not necessarily have the same characteristics as married people.

\section{CONCLUSION}

The present study found that the prevalence of dementia was different between widowed and nonwidowed people, being higher among non-widowed individuals ( $65 \%$ of cases). There was a link between widowhood and other social, demographic and clinical characteristics, showing that widowed people living with dementia tended to be older and have a lower level of schooling than the non-widowed. Widowed patients were older at the time of the onset of symptoms, and had a higher CDR score, as well as lower MMSE score, than non-widowed patients. There was no statistical association between widowhood and the time elapsed since the start of symptoms.

There was also a certain degree of association in terms of gender, with women living with dementia having a more advanced age and poorer levels of schooling than men. Women were also older at the onset of symptoms and had lower MMSE scores and greater CDR scores than men. There was also no statistical association with time since onset of symptoms, in this case.

When analysing gender in isolation, it was found that both widows and widowers were older and had a lower level of schooling. In addition, widows and widowers were older at the onset of symptoms than non-widowed patients. There was only a difference in MMSE scores among men, with widowers having a lower score than widows. Differences were not observed in either CDR scores or time since onset of symptoms.

These differences clearly suggest a need to evaluate the impact of widowhood on widows and widowers with dementia, as the loss of a spouse can result in different outcomes. It also warns of the need to adopt health measures with a special focus, with the aim of achieving more effective prevention in cases of dementia, as well as early diagnosis and more appropriate and more efficient health care strategies. 


\section{REFERENCES}

1. Stern Y. Cognitive reserve in ageing and Alzheimer's disease. Lancet Neurol. 2012;11(11):1006-12.

2. Kuiper JS, Zuidersma M, Oude Voshaar RC, Zuidema SU, van den Heuvel ER, Stolk RP, et al. Social relationships and risk of dementia: a systematic review and meta-analysis of longitudinal cohort studies. Ageing Res Rev. 2015;22:39-57.

3. Perrig-Chiello P, Spahni S, Höpflinger F, Carr D. Cohort and gender differences in psychosocial adjustment to later-life widowhood. J Gerontol Ser B Psychol Sci Soc Sci. 2016;71(4):765-74.

4. Schulz R, Newsom JT, Fleissner K, Decamp AR, Nieboer AP. The effects of bereavement after family caregiving. Aging Ment Health.1997;1(3):269-82.

5. Sundström A, Westerlund O, Mousavi-Nasab H, Adolfsson R, Nilsson L-G. The relationship between marital and parental status and the risk of dementia. Int Psychogeriatr. 2014;26(5):749-57.

6. Bertolucci PH, Brucki SM, Campacci SR, Juliano Y. Mini-Exame do estado Mental em uma população geral: impacto da escolaridade. Arq Neuropsiquiatr. 1994;52(1):1-7.

7. Hughes CP, Berg L, Danziger WL, Coben LA, Martin RL. A New clinical scale for the staging of dementia. Br J Psychiatry.1982;140(6):566-72.

8. American Psychiatric Association. Diagnostic and statistical manual of mental disorders, 4th ed. Washington, DC: American Psychiatric Association; 1994.

9. McKhann G, Drachman D, Folstein M, Katzman R, Price D, Stadlan EM. Clinical diagnosis of Alzheimer's disease: report of the NINCDS-ADRDA Work Group under the auspices of Department of Health and Human Services Task Force on Alzheimer's Disease. Neurology. 1984;34(7):939-44.

10. Roman GC, Tatemichi TK, Erkinjuntti T, Cummings JL, Masdeu JC, Garcia JH, et al. Vascular dementia: diagnostic criteria for research studies: report of the NINDS-AIREN International Workshop. Neurology. 1993;43(2):250-60.

11. Sommerlad A, Ruegger J, Singh-Manoux A, Lewis G, Livingston $G$. Marriage and risk of dementia: systematic review and meta-analysis of observational studies. J Neurol Neurosurg Psychiatry. 2018;89(3):231-8.

12. Gerritsen L, Wang HX, Reynolds CA, Fratiglioni L, Gatz M, Pedersen NL. Influence of negative life events and widowhood on risk for dementia. Am J Geriatr Psychiatry. 2017;25(7):766-78.

13. Hughes ME, Waite LJ. Marital biography and health at mid-life. J Health Soc Behav. 2009;50(3):344-58.
14. Sundström A, Westerlund O, Kotyrlo E. Marital status and risk of dementia: a nationwide populationbased prospective study from Sweden. BMJ Open. 2016;6(1):1-7.

15. Shin SH, Kim G, Park S. Widowhood status as a risk factor for cognitive decline among older adults. Am J Geriatr Psychiatry. 2018;26(7):778-87.

16. Amieva H, Mokri H, Le Goff M, Meillon C, JacqminGadda H, Foubert-Samier A, et al. Compensatory mechanisms in higher-educated subjects with Alzheimer's disease: a study of 20 years of cognitive decline. Brain. 2014;137(4):1167-75.

17. Sundström A, Westerlund O, Mousavi-Nasab H, Adolfsson R, Nilsson LG. The relationship between marital and parental status and the risk of dementia. Int Psychogeriatr. 2014;26(5):749-57.

18. Donnellan WJ, Bennett KM, Soulsby LK. How does carer resilience change over time and care status?: A qualitative longitudinal study. Aging Ment Health. 2018;1-7.

19. Feng L, Ng XT, Yap P, Li J, Lee TS, Hakansson K, et al. Marital status and cognitive impairment among community-dwelling chinese older adults: the role of gender and social engagement. Dement Geriatr Cogn Disord Extra. 2014;4(3):375-84.

20. Hajek A, Brettschneider C, Ernst A, Posselt T, Wiese B, Prokein J, et al. Longitudinal predictors of informal and formal caregiving time in communitydwelling dementia patients. Soc Psychiatry Psychiatr Epidemiol. 2016;51(4):607-16.

21. Pradier C, Sakarovitch C, Le Duff F, Layese R, Metelkina A, Anthony S, et al. The Mini Mental State Examination at the time of Alzheimer's disease and related disorders diagnosis, according to age, education, gender and place of residence: a crosssectional study among the French National Alzheimer database. PLoS ONE. 2014;9(8):1-8.

22. Derreberry TM, Holroyd S. Dementia in women. Psychiatr Clin N Am. 2017;40(2):299-307.

23. Erol R, Brooker D, Peel E. Women and dementia: a global research review. [London]: Alzheimer's Disease International; 2015.

24. Sharma N, Chakrabarti S, Grover S. Gender differences in caregiving among family - caregivers of people with mental illnesses. World J Psychiatry. 2016;6(1):7-17.

25. Almada AZ. Gender and caregiving: a study among hispanic and non-hispanic white frail elders [tese]. Virginia: Virginia Polytechnic Institute and State University, Department of Sociology; 2016. 